Received 06/26/2021 Review began 08/24/2021 Review ended 02/24/2022 Published 03/03/2022

\section{(c) Copyright 2022}

Toor et al. This is an open access article distributed under the terms of the Creative Commons Attribution License CC-BY 4.0. which permits unrestricted use, distribution, and reproduction in any medium, provided the original author and source are credited.

\title{
Prevalence of Central Line-Associated Bloodstream Infections (CLABSI) in Intensive Care and Medical-Surgical Units
}

\author{
Harjyot Toor ${ }^{1}$, Saman Farr ${ }^{1}$, Paras Savla ${ }^{1}$, Samir Kashyap ${ }^{1}$, Sharon Wang ${ }^{2}$, Dan E. Miulli ${ }^{1}$ \\ 1. Neurosurgery, Riverside University Health System Medical Center, Moreno Valley, USA 2. Infectious Disease, \\ Arrowhead Regional Medical Center, Colton, USA
}

Corresponding author: Harjyot Toor, harjyot.toor@tu.edu

\begin{abstract}
Background: Central line-associated bloodstream infections (CLABSIs) remain an important preventable healthcare-associated infection with a 2020 rate of 0.87 (per 1,000 central line days) in the United States intensive care units (ICU).
\end{abstract}

Methods: This was a retrospective cohort study of all adult patients in our institution. The total number of central venous catheter (CVC) insertions and line days were determined using daily unit logs maintained by unit managers. Central line insertion practice (CLIP) compliance was calculated as the total number of CLIP forms submitted divided by the total number of newly-inserted CVCs with and without associated CLIP forms as determined by unit logs.

Results: A total of 1,125 CVCs were reviewed (448 - ICU and 677 - medical-surgical units). Of the 13 CLABSI, one patient had internal jugular (IJ), one patient had subclavian (SC), four patients had femoral, three patients had peripherally inserted central catheter (PICC) and four patients had hemodialysis catheters. Patients with CLABSI had CVC inserted for a range of five to 92 days with the average number of line days being 29 days.

Conclusion: Based on the analysis of our CLABSI patient population, we recommend our institution implement the following criteria to decrease the prevalence of CLABSI: All patients receiving a CVC must adhere to CLIP documentation in all units, any femoral or HD CVC placed without a CLIP form should have the line changed within 48 hours, those patients with a femoral CVC or hemodialysis catheter in place for four days or greater with an abnormal WBC $(<4.0 \mathrm{or}>11 \mathrm{mg} / \mathrm{dL})$ or abnormal temperature $(<97.0 \mathrm{~F}$ or $>100.4 \mathrm{~F})$ should be considered for catheter exchange, and those patients with an IJ, SC, or PICC CVC in place for seven days or greater with an abnormal WBC or abnormal temperature should have the catheter changed.

Categories: Infectious Disease, Neurosurgery, Trauma

Keywords: clabsi, central line, critical care, infectious disease, trauma

\section{Introduction}

Central line-associated bloodstream infections (CLABSIs) remain an important, preventable healthcareassociated infection with a 2020 rate of 0.87 (per 1,000 central line days) in the United States intensive care units (ICU) [1-3]. CLABSIs carry a mortality rate of $12 \%-15 \%$ and an odds ratio of in-hospital death is as high as 2.75 [4]. CLABSIs are also associated with increased length of hospital stay and increased healthcare cost with each case accounting for approximately $\$ 46,000$. Most cases of CLABSIs are preventable with proper aseptic techniques, surveillance, and management strategies.

The CDC's “Guidelines for the Prevention of Intravascular Catheter-Related Infections” advocate evidencebased central line insertion practices (CLIPs) to reduce the risk of subsequent CLABSI. The sterile technique includes hand hygiene, maximal sterile barriers during insertion, use of an antiseptic prior to insertion with time to allow the antiseptic to dry before catheter insertion (CDC NHSN). Centers across the United States have implemented CLIP and CLIP adherence is under stringent institutional and federal supervision. Our goals were to: (1) assess the prevalence of CLABSI in both ICU and medical-surgical units (also known as the "floor") at our institution and (2) assess whether CLIP sheet compliance decreases the incidence of CLABSI.

\section{Materials And Methods}

This was a retrospective cohort study of all adult patients in our institution, a Level 2 trauma center in Southern California, with an indwelling central venous catheter (CVC) of two or more days between January 1, 2020 and December 31, 2020. All patients were screened with ICD-10 Code T80.211A (CLABI) and those submitted by our institution to the National Healthcare Safety Network (NHSN). 


\section{Cureus}

CLABSI was defined as a laboratory-confirmed new bloodstream infection that is not secondary to an infection at another body site - in accordance with the CDC definition. Patient data were collected from unit $\log$ review and from the log submitted by our institution to the National Healthcare Safety Network (NHSN). Patients under 18 years, having a CVC placed prior to admission, negative blood cultures, open wounds/abrasions within $10 \mathrm{~cm}$ of CVC insertion, and evidence of sepsis and/or infection on admission were excluded. CVC documentation was reviewed in physical and electronic charts. Visual inspection and charted data on insertion date, body site location, and line type were compared to nursing electronic and paper CVC assessments. Patients missing any or all these metrics were excluded from the study.

Multiple attempts were made to collect from the electronic medical record the total number of CVC insertions performed at our institution using CPT codes and ICD-10 procedure codes but did not yield results. The total number of CVC insertions and line days were eventually determined using daily unit logs maintained by unit managers. CLIP compliance was calculated as the total number of CLIP forms submitted divided by the total number of newly inserted CVCs with and without associated CLIP forms as determined by unit logs. For the latter, missing forms were considered non-compliant. Comparisons were made using chi-squared analysis. Data were stratified by ICU/non-ICU location and CVC-type (subclavian [SC], internal jugular [IJ], femoral, peripherally inserted central catheter [PICC], or hemodialysis [HD]).

\section{Results}

\section{CLABSI prevalence}

A total of 1,125 CVC were reviewed (448 from ICU and 677 from medical-surgical units). Of those 1,125 central lines; 220 were IJ, 107 were SC, 188 were femoral, 449 were PICC, and 161 were HD catheters (Table 1). Fifty patients were diagnosed and treated for CLABSI at the time of discharge. Some patients were included more than once as they had multiple, separate hospitalizations. Thirty-two patients were excluded due to pre-hospital CVC insertion, one patient was excluded due to no growth on blood culture and four patients were excluded due to positive blood culture prior to two days (Tables 2, 3). The total number of days of exposure to CVCs (line days) among all patients with CLABSI was 379 days, ranging from five to 93 days. Of the 13 CLABSI, one patient had IJ, one patient had SC, four patients had femoral, three patients had PICC, and four patients had hemodialysis catheters (Table 4). CLABSI rate for the ICU was 1.7 (per 1,000 central line days) and for the medical-surgical units (or floor units) was 2.8 (per 1,000 central line days) (Figure 1). The highest incidences of CLABSI by type of CVC are femoral and hemodialysis (Table 4). When looking at the average line days to infection, it was 23.25 days for a CLABSI in the ICU and 31.6 days in the medical-surgical unit. When looking at the average line days without infection it was 5.3 days for a CVC without CLABSI in the ICU and 5.34 days in the medical-surgical unit (Table 5).

\begin{tabular}{|c|c|c|c|c|c|c|c|}
\hline Central Lines Placed 2020 & Internal Jugular & Subclavian & Femoral & PICC & Hemodialysis & Total & Line Days \\
\hline ICU & 173 & 35 & 169 & 70 & 1 & 448 & 2,373 \\
\hline Medical Surgical & 47 & 72 & 19 & 379 & 160 & 677 & 3,617 \\
\hline Total & 220 & 107 & 188 & 449 & 161 & 1,125 & 5,990 \\
\hline
\end{tabular}

\section{TABLE 1: Type of central venous catheter insertion in ICU and medical-surgical patients}

PICC - peripherally inserted central catheter

Patients with central line-associated bloodstream infections (CLABSIs)

Screened for hospital acquired CLABSI

Excluded due to line placement prior to admission

Negative blood cultures

Positive blood culture prior to line placement

TABLE 2: Screening for CLABSI 


\section{Cureus}

\begin{tabular}{|c|c|c|c|c|c|c|c|}
\hline Patient & Line type & $\begin{array}{l}\text { \# of line } \\
\text { days }\end{array}$ & $\begin{array}{l}\text { Temp } 2 \text { days before }(+) \\
\text { blood } \mathrm{cx}\end{array}$ & $\begin{array}{l}\text { Temp } 1 \text { days before }(+) \\
\text { blood cx }\end{array}$ & Tmax & WBC & Organism \\
\hline 1 & Hemodialysis & 19 & 99.8 & 99.1 & 99.6 & 16.3 & Proteus mirabilis \\
\hline 2 & Hemodialysis & 92 & 98.4 & 99.8 & 102.9 & 27 & $\begin{array}{l}\text { Staphylococcus } \\
\text { epidermidis }\end{array}$ \\
\hline 3 & Femoral & 36 & 97.6 & 97.5 & 97.8 & 13.6 & $\begin{array}{l}\text { Candida } \\
\text { orthopsilosis }\end{array}$ \\
\hline 4 & Subclavian & 18 & 98.7 & 98.5 & 99.6 & 3.2 & $\begin{array}{l}\text { Enterococcus } \\
\text { faecalis }\end{array}$ \\
\hline 5 & Hemodialysis & 28 & 98.8 & 98.7 & 100.6 & 20 & $\begin{array}{l}\text { Pseudomonas } \\
\text { aeruginosa }\end{array}$ \\
\hline 6 & PICC & 8 & 98.9 & 99.8 & 101.2 & 0.2 & Escherichia coli \\
\hline 7 & Femoral & 8 & 98.4 & 98.2 & 98.7 & 14.6 & $\begin{array}{l}\text { Enterococcus } \\
\text { faecalis }\end{array}$ \\
\hline 8 & Femoral & 11 & 100.4 & 100.5 & 104 & 34.3 & $\begin{array}{l}\text { Staphylococcus } \\
\text { aureus }\end{array}$ \\
\hline 9 & IJ & 27 & 98.9 & 99.8 & 101.2 & 7.3 & $\begin{array}{l}\text { Staphylococcus } \\
\text { epidermis }\end{array}$ \\
\hline 10 & Hemodialysis & 5 & 97.5 & 99 & 99.7 & 3.9 & $\begin{array}{l}\text { Enterococcus } \\
\text { faecalis }\end{array}$ \\
\hline 11 & $\begin{array}{l}\text { Right } \\
\text { Femoral }\end{array}$ & 40 & 99.9 & 100.1 & 102.5 & 16.3 & Proteus mirabilis \\
\hline 12 & PICC Line & 47 & 98.9 & 99.8 & 101.2 & 0.2 & Escherichia Coli \\
\hline 13 & PICC Line & 37 & 98.7 & 98.5 & 103.2 & 9.7 & $\begin{array}{l}\text { Staphylococcus } \\
\text { aureus }\end{array}$ \\
\hline
\end{tabular}

TABLE 3: Demographics of patients meeting criteria for hospital-acquired CLABSI

IJ - internal jugular; PICC - peripherally inserted central catheter; CLABSI - central line-associated bloodstream infection

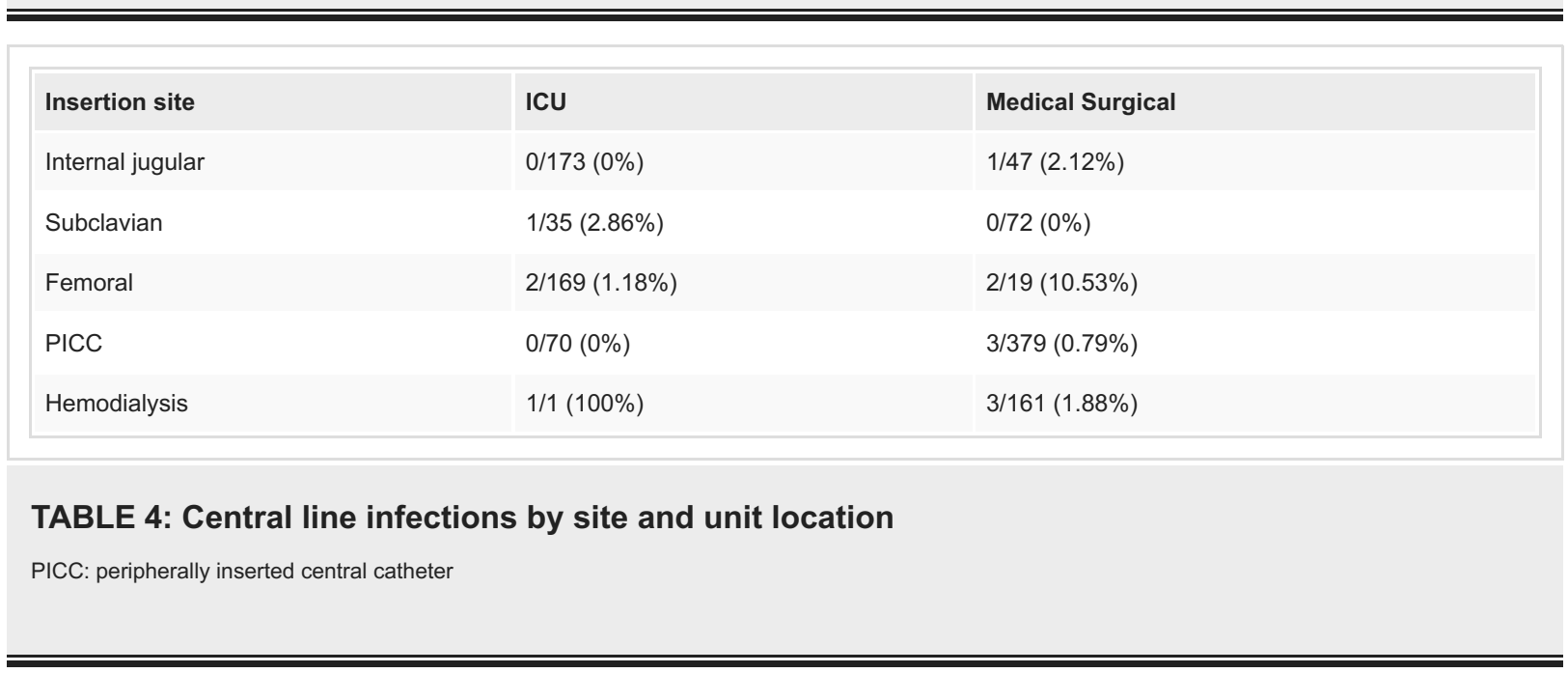




\section{Cureus}

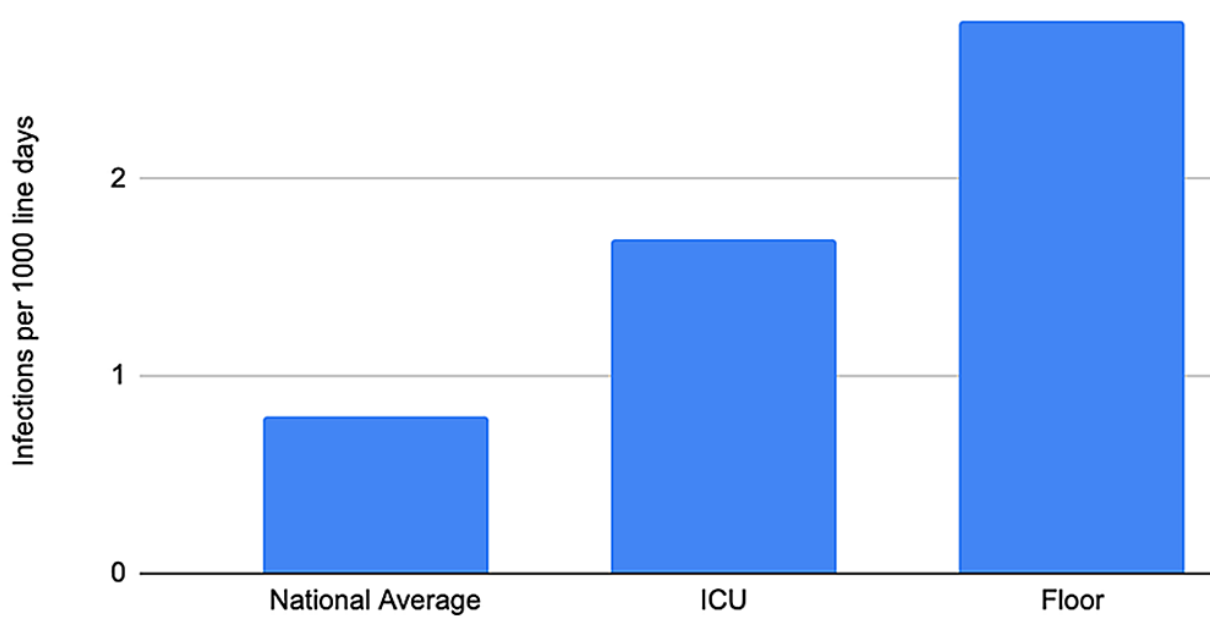

FIGURE 1: Institutional comparison of CLABSI rate to the national average

Floor: Medical-Surgical Unit, CLABSI - Central line-associated bloodstream infection

\begin{tabular}{|l|l|l}
\hline \# of CVC & Line days (mean) & Line days (total)
\end{tabular}

ICU

CLABSI

4

23.25

93

No CLABS

444

5.3

2,373

Medical Surgical

CLABSI

9

31.66

284

No CLABSI

668

5.3

3,617

\section{TABLE 5: Line days in patients with and without CLABSI}

CVC - central venous catheter, CLABSI - central line-associated bloodstream infection

Patients with CLABSI had CVC inserted for a range of five to 92 days with the average number of line days being 29 days. The average temperature maximum for the patients with CLABSI was $101.1 \mathrm{~F}$ with $64.3 \%$ of patients having a temperature greater than 100.4F. Normal number of white blood cells (WBC), defined as 4,500 to 11,000 WBCs per microliter, $92.3 \%$ of patients (12 of 13) had an abnormal WBC. Twelve of 13 patients were diagnosed with bacteremia (4/12 gram-positive, $8 / 12$ gram-negative), while one was found to have Candida (Table 3).

\section{CLIP compliance}

Among all CVCs inserted, 58.6\% (660/1125) had CLIP forms submitted. The rate of CLABSI from those CVC inserted with CLIP forms submitted was $61.5 \%(8 / 13)$.

\section{Discussion}

In the present study, the rate of CLABSI in both the intensive care and medical-surgical units was 1.7 per 1,000 central line days and 2.8 per 1,000 central line days, respectively. This is triple the CLABSI rate in 2019, which was 0.69 per 1,000 line days. In 2020 during the COVID pandemic, the national average increased to 0.87 per 1,000 central line days in the ICU [1-3]. When accounting for all CVCs inserted at our institution (emergency department, operating room, interventional radiology, ICU, or floor units) rather than only those CVCs with submitted CLIP forms, the rate of CLABSI was significantly higher. The incidence CLABSI with CLIP form was $61.5 \%$, and the incidence of CLABSI without CLIP form was $38.4 \%$. 
A higher prevalence of CLABSI was found with femoral and HD CVCs. When these were removed, the infection rate adjusted to 0.9 per 1,000 central line days - in line, on par with the national average. Femoral lines are well known to have higher rates of CLABSI, while SC lines are the least at risk for infection [5-8]. Furthermore, the CDC has recommended against the use of femoral central lines whenever possible in adult patients (Level of evidence: IA, BSI guidelines) and has recommended the use of the SC site in nonhemodialysis patients for short-term infusion (Level of evidence: IB) [9]. The preferred site for tunneled catheters has not been established, but the SC site should be avoided as hemodialysis patients with SC sites are at a higher risk of developing SC vein stenosis [9].

Concurrent CVC use is associated with nearly twofold risk of CLABSI compared with use of a single CVC [2]. Dube et al. reviewed 6877 patients with two or more days with concurrent or subsequent CVCs and found that 74 of 3,932 patients with concurrent CVC use (1.9\%) developed CLABSI, compared with 81 of 7,864 patients with single CVC use (1.0\%) [2]. Having two CVCs for longer than two-thirds of a patient's CVC use duration was associated with increased likelihood of developing a CLABSI, adjusting for central line days and comorbidities. These lines were most frequently indicated for nutrition (14.1\% of patients) or hemodialysis (43.4\% of patients) [2].

The CLABSI rate was higher in patients in which CLIP forms were used (61.5\%) compared to patients in which they were not used (38.4\%). CLIP monitoring failed to capture $41.4 \%$ of CVCs inserted in our institution, $10.4 \%$ higher than estimated figures bythe National Healthcare Safety Network [10]. Noncompliance with CLIP form submission could be a source of preventable infection as it is associated with poor insertion practices and higher infection [10]. Although the rate of CLABSIs was higher in the CLIP form group, this is probably due to the fact that the infections were more appropriately documented.

Our institution's poor CLIP form compliance was suggested to be due to the high volume of trauma cases and the emergent conditions under which CVCs are placed to resuscitate and stabilize trauma patients. Major trauma centers have been found to have higher rates of CLABSI [11]. In such cases, although adherence to best insertion practices may be difficult it must be required. In those patients with missing CLIP forms, we cannot assume that proper sterile technique was followed. Consequently, CVC without CLIP form compliance should be deemed a risk factor for CLABSI and these patients should have the CVC changed within 48 hours. When catheters are inserted under emergent conditions without CLIP forms, and the use of sterile technique cannot be ensured, the CDC recommends replacement of the catheter within 48 hours [9].

Proper documentation with checklists to ensure adherence to evidence-based guidelines has been shown to decrease CLABSI rates [12,13]. Failure to account for our institution's non-compliance with CLIP forms may overestimate adherence to CLIP and its role in reducing CLABSI. Identifying low compliance with CLIP forms at our hospital highlights an area where significant improvements can be made to increase proper sterile insertion practices. This effort should be multidisciplinary, involving healthcare providers, infection control staff, nurses and unit managers. When stratified by line type, form submission, and adherence to CLIP elements, there is twofold higher compliance among PICC insertions [10]. Scott et al. found PICCs had more reliable line-day documentation. This was likely the effect of an optimized approach to insertion by a dedicated PICC team whereby CLIP form completion and insertion elements are included in the standardized protocol [10]. By contrast, all non-PICC lines were placed by resident or attending physicians who receive standardized insertion education, but are not necessarily required to ensure proper completion of this [10]. Quan et al. showed that CLIP form use and documentation regarding central line days improved from $55 \%$ to $99 \%$ when an electronic health record system was used to streamline workflow and CLIP elements were embedded into an electronic procedure note [14].

Based on the analysis of our CLABSI patient population, we suggest that the following changes could potentially help to decrease the rate of CLABSI: (1) that all patients receiving a CVC should adhere to CLIP documentation in all units, (2) that any femoral or HD CVC placed without a CLIP form should have the line changed within 48 hours, (3) that those patients with a femoral CVC or hemodialysis catheter in place for four days or greater with an abnormal WBC $(<4.0$ or $>11 \mathrm{mg} / \mathrm{dL})$ or abnormal temperature ( $<97.0 \mathrm{~F}$ or $>100.4 \mathrm{~F})$ should be considered for catheter exchange, and (4) that those patients with an IJ, SC, PICC CVC in place for seven days or greater with an abnormal WBC or abnormal temperature should have the catheter changed.

Future areas of study will include evaluation of line care practices given the average number of line days in patients with CLABSI. Another potential area to evaluate will be the duration that peripheral lines are in place at the time of a CLABSI.

\section{Limitations}

Our institutional review carried limitations. The biggest limitation was the inability to verify the total handwritten logged number of lines placed in the hospital with the electronic medical record system. Multiple attempts were made to collect the total number of CVC insertions performed at our institution using CPT codes and ICD-10 Procedure Codes, but this did not yield usable data. The total number of CVC insertions and line days were eventually determined using daily unit logs maintained by the unit managers. These unit logs proved to be highly accurate as they are maintained and updated daily by the unit managers. However, this limitation also makes it difficult to access in real-time CVC insertion and CLABSI to quickly 


\section{Cureus}

improve line care practices.

\section{Conclusions}

Our institutional review found a rate of CLABSI twice the national average in the ICU and triple the average in patients on the floor. We found poor compliance with CLIP form completion along with a higher prevalence in those with femoral and hemodialysis catheters. We propose a new set of criteria to reduce the number of CLABSI at our institution.

\section{Appendices}

THIS FORM MUST BE COMPLETED FOR EACH CENTRAL LINE INSERTION OBSERVER MUST ENSURE ALL SECTIONS OF THIS FORM ARE COMPLETE

Patient Room Number: $\square$ Oupatient $\square E D$ IIR $\square O R$

Assigned Service of Inserter: $\square$ Anesthesia $\square$ Emergency $\square$ Family $\square$ Internal Medicine $\square$ Neurology $\square$ Neurosurgery $\square$ Surgery $\square$ IR Occupation of Inserter: $\square$ Attending Physician $\square$ Fellow $\square$ ResidentIIntern $\square$ Physician Assistant $\square$ PICC* Line Nurse $\square$ Other Key: PICC = Peripherally Inserted Central Catheter

Reason for Insertion: $\square$ New Indication for Central Line $\square$ Replace Melfunctioning Central Line $\square$ Suspected CLABSI* $\square$ Other Key: CLABSI = Central Line-Associated Bloodstream Stream Infection

Central Line Type: $\square$ Central Venous Catheter (non-tunneled, non-dialysis) $\square$ Dialysis, Non-Tunneled $\square$ Dialysis, Tunneled $\square$ Porta-Cath (tunneled, i.e. Hickman, Broviac) $\square$ PICC $\square$ Trialysis Catheter $\square$ Other

Insertion Site: $\square$ Internal Jugular $\square$ Subclavian $\square$ Umbilical $\square$ Upper Extremity (PICC) $\square$ Scalp $\square$ Femoral - If femoral is the optimal site, reason:

"If exchanging or replacing line, was it: $\square$ N/A $\square$ Exchanged over J-Wire $\square$ Fresh Stick

Pre-Procedure:

Order completed - Purpose of line

$\square$ Informed Consent Obtained (Not required for PICC)

$\square$ Ensure room is clean (contact EVs if needed)

$\square$ Coagulation Panel (if applicable)

$\square$ Patent Assessment - Allergies, meds, contraindications, lab results

(if required). If family present, explain procedure/educate

$\square$ Gather Supplies/Multi-line Cart and Ultrasound Machine

$\square$ Ensurc availability of an observer for the procedure

TIMEOUT REQUIRED (Observer calls Timeout)
Observer reads consent out loud confirming:
$\square$ Patient Name \& Date of Birth
$\square$ Correct Procedure/Site
$\square$ Measure arm circumference, Mark Site (for PICC line)
Prep Patient
$\square$ All staft in the room performs Hand Hygiene
$\square$ Ensure ready access to supplies / ultrasound / monitor
$\square$ Administer medications as ordered
$\square$ Open kit on clean surface

Procedure: It is the responsibility of all staff members who are involved in the line insertion to ensure that each step is performed correctly. Any member of the team has the authority to stop the procedure if a break in technique is observed; the inserter must stop the procedure and make the neccessary corrections before proceeding.

$\square$ Inserter dons clean gloves, cap and imask

Skin Prep:

$\square$ CHG・ $\square$ Povidone iodine $\square$ Alcohol

$\square$ Scrub with CHG in a back and forth metion per manufacturer recommender time frame.

$\square$ CHG IS COMPLETELY dry at time of first skin puncture

Key: $C H G$ a Chlorhexidine gluconate

$\square$ Inserter performs Hand Hygiene \& dons sterile gloves \& gown

$\square$ Place full body drape on patient

$\square$ Local Anesthesia (if indicated)

$\square$ After 3 unsuccessful attempts, use more

oxperienced Provider.

$\square$ Aspirate each lumen for blood return $\square$ Flush each lumen for patency

$\square$ Central Line socured in place at $\mathrm{cm}$ Apply Dressing

$\square$ Site Cleansed with $\mathrm{CHO}$

$\square$ Apply Biopatch (blue side to the sky)

$\square$ Place dressing on patient

$\square$ Date, time, and initial the central line dressing

Post Procedure Instructions

$\square$ Dispose of Sharps/Meds/PPE

$\square$ Chest X-Ray Done

$\square$ Document Procedure Note, including purpose for line

$\square$ Clean cart and ultrasound with antimicrobial wipes after procedure call SPD to do a multiline cart exchange

$\square$ Completed Cllp Form Faxed to Epidemiolegy at 03180

$\square$ Place this completed form in Progress Note section of the Patient Medical Record

$\square$ If CXR indicates repositioning is necessay, document in Progress Notes when completed.

$\square$ Practitioner's Order received that line is $O K$ to use after Chest $X$.ray

Was this insertion successful? $\square$ Yes $\square$ No If not, a new CLIP form must be completed for each subsequent attempt

REQUIRED DOCUMENTATION:

Post Central line: Guidewire removed and is intact. WITNESSED BY BOTH INSERTER AND OBSERVER $\square$ Yes $\square$ No

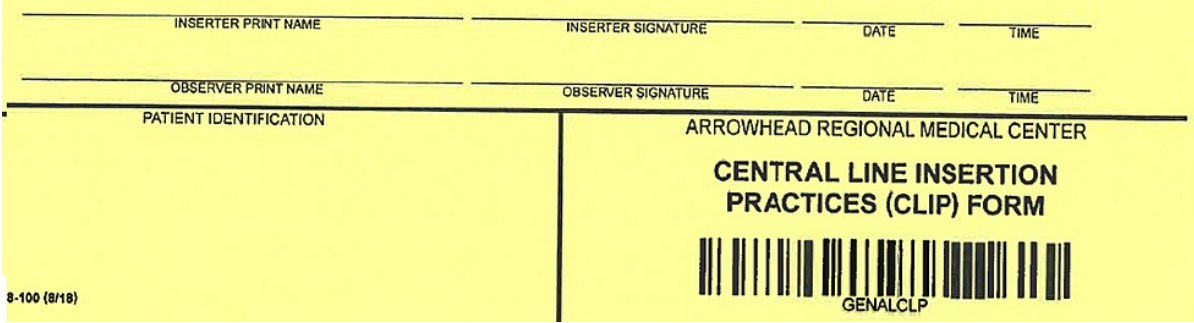

FIGURE 2: Institutional CLIP form

\section{Additional Information}

Disclosures

Human subjects: All authors have confirmed that this study did not involve human participants or tissue. Animal subjects: All authors have confirmed that this study did not involve animal subjects or tissue. Conflicts of interest: In compliance with the ICMJE uniform disclosure form, all authors declare the 
following: Payment/services info: All authors have declared that no financial support was received from any organization for the submitted work. Financial relationships: All authors have declared that they have no financial relationships at present or within the previous three years with any organizations that might have an interest in the submitted work. Other relationships: All authors have declared that there are no other relationships or activities that could appear to have influenced the submitted work.

\section{References}

1. Haddadin Y, Annamaraju P, Regunath H: Central Line Associated Blood Stream Infections. StatPearls Publishing, Treasure Island, FL; 2021.

2. Dube WC, Jacob JT, Zheng Z, Huang Y, Robichaux C, Steinberg JP, Fridkin SK: Comparison of rates of central line-associated bloodstream infections in patients with 1 vs 2 central venous catheters. JAMA Netw Open. 2020, 3:e200396. 10.1001/jamanetworkopen.2020.0396

3. Patel PR, Weiner-Lastinger LM, Dudeck MA, et al.: Impact of COVID-19 pandemic on central-lineassociated bloodstream infections during the early months. Infect Control Hosp Epidemiol. 2021, 1-4. 10.1017/ice.2021.108

4. Ziegler MJ, Pellegrini DC, Safdar N: Attributable mortality of central line associated bloodstream infection: systematic review and meta-analysis. Infection. 2015, 43:29-36. 10.1007/s15010-014-0689-y

5. Pitiriga V, Kanellopoulos P, Bakalis I, Kampos E, Sagris I, Saroglou G, Tsakris A: Central venous catheterrelated bloodstream infection and colonization: the impact of insertion site and distribution of multidrugresistant pathogens. Antimicrob Resist Infect Control. 2020, 9:189. 10.1186/s13756-020-00851-1

6. Akhter M, Runde D, Lee J: Which central line insertion site is the least prone to infection? . Ann Emerg Med. 2013, 61:362-3. 10.1016/j.annemergmed.2012.10.002

7. Buetti N, Mimoz O, Mermel L, et al.: Ultrasound guidance and risk for central venous catheter-related infections in the ICU. A post hoc analysis of individual data of three multi-centric randomized trials. Clin Infect Dis. 2021, 73:e1054-61. 10.1093/cid/ciaa1817

8. Merrer J, De Jonghe B, Golliot F, et al.: Complications of femoral and subclavian venous catheterization in critically ill patients: a randomized controlled trial. JAMA. 2001, 286:700-7. 10.1001/jama.286.6.700

9. Guidelines for the prevention of intravascular catheter-rlated infections . (2020). Accessed: June 22, 2021: https://www.cdc.gov/infectioncontrol/guidelines/bsi/index.html.

10. Scott SK, Gohil SK, Quan K, Huang SS: Marked reduction in compliance with central line insertion practices (CLIP) when accounting for missing CLIP data and incomplete line capture. Am J Infect Control. 2016, 44:242-4. 10.1016/j.ajic.2015.09.007

11. Kaminski MA, Episcopia B, Malik S, Fornek M, Landman D, Xavier G, Quale J: Trends in central-lineassociated bloodstream infections and catheter-associated urinary tract infections in a large acute-care hospital system in New York City, 2016-2019. Infect Control Hosp Epidemiol. 2021, 42:842-6. 10.1017/ice.2020.1293

12. Marschall J, Mermel LA, Classen D, et al.: Strategies to prevent central line-associated bloodstream infections in acute care hospitals. Infect Control Hosp Epidemiol. 2008, 29 Suppl 1:S22-30. 10.1086/591059

13. Berenholtz SM, Pronovost PI, Lipsett PA, et al.: Eliminating catheter-related bloodstream infections in the intensive care unit. Crit Care Med. 2004, 32:2014-20. 10.1097/01.ccm.0000142399.70913.2f

14. Quan KA, Cousins SM, Porter DD, et al.: Electronic health record solutions to reduce central line-associated bloodstream infections by enhancing documentation of central line insertion practices, line days, and daily line necessity. Am J Infect Control. 2016, 44:438-43. 10.1016/j.ajic.2015.10.036 